\title{
Urinary incontinence - treatment
}

\section{Therapie der Stressinkontinenz}

Für die Behandlung der Stressinkontinenz stehen konservative Therapie, Operationsverfahren und Hilfsmittelversorgung zur Verfügung. Diese Therapieoptionen kommen oft auch gemeinsam zum Einsatz. Welche Therapie gewählt wird, hängt vom Allgemeinzustand der Patientin, von der Funktion des Verschlussorgans und dem Ausmaß der Beckenbodeninstabilität ab.

\section{Konservative Therapie}

Bei Stressinkontinenz wird v.a. Beckenbodentraining eingesetzt. Dabei haben sowohl Motivation und Compliance der Betroffenen als auch die fachkundige Anleitung große Bedeutung. In weiteren Schritten sollten Biofeedback-Verfahren und Elektrostimulation einbezogen werden. Berücksichtigen sollte man, dass frühestens nach 4-6 Monaten intensiver Physiotherapie ein Effekt abzuschätzen ist $(16,8)$.

\section{Medikamentöse Therapie}

Der Schließmuskelapparat ist pharmakologisch nur bei leichter bis mittelschwerer Stressinkontinenz erfolgversprechend beeinflussbar. Zu nennen sind hier neben Östrogen $\alpha$-Adrenergika sowie Antidepressiva. In der Grundlagenforschung werden zur Zeit noch andere Stoffe untersucht (13). Die Östrogentherapie stellt seit Jahren den Hauptanteil der medikamentösen Therapieverfahren dar. Der Effekt basiert auf einer Proliferation des Harnröhrenepithels, einer submukösen Kongestion und einer gesteigerten Sensibilität von $\alpha$ Rezeptoren, die auf endogene Katecholamine zugeschnitten sind. So kann es zu einer Rückbildung z.B. der postmenopausalen atrophischen Urethritis kommen. In allen relevanten Untersuchungen der letzten Jahre war eine niedrig dosierte vaginale Östrogentherapie einer höher dosierten systemischen Behandlung überlegen (14).

\section{Operative Therapie}

Erst wenn die konservativen Verfahren ausgeschöpft sind, über einen adäquaten Zeitraum zum Einsatz kamen und erfolglos blieben, sollte unter Berücksichtigung der individuellen Situation eine Operation in Betracht gezogen werden. Standardverfahren wie die Kolposuspensi- on und Schlingenoperationen haben ihren Stellenwert. Jedoch wurden in den letzten Jahren schonendere Eingriffe wie das TVT-Verfahren („tension free vaginal tape“) in Schweden entwickelt (17).

\section{Hilfsmittel}

Bei Persistenz der Beschwerdesymptomatik und/oder für einen kurzfristigen Einsatz gibt es verschiedene Hilfsmittel (von der Pessarbehandlung bis zu verschiedensten Harnröhrenstöpseln sowie modifizierter Tamponbenutzung). Diese sollte man unter Berücksichtigung der Einstellung der Patientin und Indikation anwenden (10).

\section{Therapie der Urge-(Drang)inkontinenz}

Bei Dranginkontinenz können durch Aufdeckung von Ursachen und einfache nicht-medikamentöse Therapie beachtliche Therapieerfolge erzielt werden. Ein Infekt der ableitenden Harnwege sollte immer ausgeschlossen oder therapiert werden. Medikamente, die eine Dranginkontinenz auslösen oder verstärken können, sollten auf ihre Indikation überprüft und gegebenenfalls abgesetzt werden.

Der Schweregrad der Dranginkontinenz hängt von 3 Faktoren ab (6):

- Intensität des Dranges

- Wegstrecke bis zur Toilette

- Fortbewegungsgeschwindigkeit des Patienten

Leichte Formen der Dranginkontinenz können also auch durch eine Toilette in angemessener Entfernung oder durch eine Verbesserung der Beweglichkeit des Patienten (z.B. durch Krankengymnastik) behandelt werden.

\section{Verhaltenstherapie}

Eine deutliche Verbesserung der Beschwerden kann durch ein Kontinenztraining (Blasentraining) erfolgen $(5,11)$. Im einfachsten Fall wird der Patient bei konstanter Trinkmenge dazu angehalten die Toilette auch ohne Harndrang regelmäßig aufzusuchen (z.B. alle 2

${ }^{1}$ Zentrum für Innere Medizin, Kliniken St. Antonius, Wuppertal

${ }^{2}$ Klinik für geriatrische Rehabilitation, Kliniken St. Antonius, Wuppertal 
Tab.1 Pharmakologische Therapie der Dranginkontinenz.

\begin{tabular}{|c|c|c|c|c|}
\hline Wirkstoff & $\begin{array}{l}\text { Minimale und maxi- } \\
\text { male Dosierung }\end{array}$ & Wirkmechanismus & Nebenwirkungen & Kontraindikationen \\
\hline $\begin{array}{l}\text { Oxybutynin-HCL } \\
\text { (Dridase }^{\circledR}, \text { Cystonorm }^{\circledR}, \\
\text { Ryol }^{\circledR}, \text { Spasyt }^{\circledR} \text { ) }\end{array}$ & $\begin{array}{l}2 \times 2,5 \mathrm{mg} \text { tägl. } \\
\text { bis } 4 \times 5 \mathrm{mg} \text { tägl. }\end{array}$ & $\begin{array}{l}\text { muskelrelaxierend, } \\
\text { lokalanästhetisch }\end{array}$ & $\begin{array}{l}\text { Mundtrockenheit (78\%), (11\%), } \\
\text { gastrointestinale Beschwerden (40\%) }\end{array}$ & $\begin{array}{l}\text { Glaukom, Myasthenia gravis, } \\
\text { Obstruktion der ableitenden } \\
\text { Harnwege, Darmobstruktion }\end{array}$ \\
\hline $\begin{array}{l}\text { Tolterodine } \\
\text { (Detrusitol }^{\circledR}, \\
\left.\text { Detrusitol retard }{ }^{\circledR}\right)\end{array}$ & $\begin{array}{l}2 \times 1 \mathrm{mg} \text { tägl. } \\
\text { bis } 4 \times 2 \mathrm{mg} \text { tägl. }\end{array}$ & antimuskarinerg & $\begin{array}{l}\text { Mundtrockenheit (40\%), Sehstörungen } \\
(5 \%), \text { Kopfschmerzen, Unwohlsein (7\%), } \\
\text { gastrointestinale Beschwerden (26\%) }\end{array}$ & $\begin{array}{l}\text { Glaukom, Myasthenia gravis, } \\
\text { Obstruktion der ableitenden } \\
\text { Harnwege, Darmobstruktion }\end{array}$ \\
\hline $\begin{array}{l}\text { Propiverinhydrochlorid } \\
\text { (Mictonetten }^{\circledR} \\
\text { Mictonorm }^{\circledR} \text { ) }\end{array}$ & $\begin{array}{l}2 \times 15 \mathrm{mg} \text { tägl. } \\
\text { bis } 4 \times 15 \mathrm{mg} \text { tägl. }\end{array}$ & antimuskarinerg & $\begin{array}{l}\text { Mundtrockenheit, gastrointestinale } \\
\text { Beschwerden, Blutdruckabfall, } \\
\text { Schwindel }\end{array}$ & $\begin{array}{l}\text { Glaukom, Myasthenia gravis, } \\
\text { Obstruktion der ableitenden } \\
\text { Harnwege, Darmobstruktion }\end{array}$ \\
\hline $\begin{array}{l}\text { Trospiumchlorid } \\
\text { (Spasmex }^{\circledR}, \text { Spasmolyt } \\
\text { Trospi }^{\circledR} \text {, }\end{array}$ & 2-4 × 10-15 mg tägl. & antimuskarinerg & $\begin{array}{l}\text { Mundtrockenheit, Sehstörungen, } \\
\text { Kopfschmerzen, Unwohlsein, } \\
\text { Obstipation }\end{array}$ & $\begin{array}{l}\text { Glaukom, Myasthenia gravis, } \\
\text { Obstruktion der ableitenden } \\
\text { Harnwege, Darmobstruktion }\end{array}$ \\
\hline $\begin{array}{l}\text { Imipraminhydrochlorid } \\
\text { (Tofranil }^{\circledR} \text {, Pryleugan }{ }^{\circledR} \text { ) }\end{array}$ & $\begin{array}{l}4 \times 25 \mathrm{mg} \text { tägl. } \\
\text { bis } 4 \times 75 \mathrm{mg} \text { tägl }\end{array}$ & $\begin{array}{l}\text { Trizyklisches } \\
\text { Antidepressivum, } \\
\text { anticholinerg, adrenerg, } \\
\text { antihistaminerg }\end{array}$ & $\begin{array}{l}\text { Mundtrockenheit, Sehstörungen, } \\
\text { Kopfschmerzen, Unwohlsein, } \\
\text { Obstipation, Leberfunktionsstörungen, } \\
\text { Bluthochdruck, Herzkreislaufstörungen }\end{array}$ & $\begin{array}{l}\text { Glaukom, Darmobstruktion, } \\
\text { Myasthenia gravis, } \\
\text { Herzrhythmusstörungen }\end{array}$ \\
\hline $\begin{array}{l}\text { Desmopressin } \\
\left(\text { Minirin }^{\circledR}\right)\end{array}$ & $\begin{array}{l}20-40 \mu \mathrm{g} \text { vor dem } \\
\text { Schlafen }\end{array}$ & Antidiuretikum & $\begin{array}{l}\text { Kopfschmerzen, Übelkeit, Reizung der } \\
\text { Nasenschleimhaut, Flush }\end{array}$ & $\begin{array}{l}\text { KHK, Bluthochdruck, Epilepsie, } \\
\text { Herzinsuffizienz }\end{array}$ \\
\hline
\end{tabular}

Stunden). Ziel ist es, das Miktionsintervall auf ca. 3-4 Stunden zu verlängern. Effektiver, aber auch aufwändiger, ist ein ambulantes oder sogar stationäres Training in einem geeigneten Zentrum. Neben der geregelten Miktion kommen hier Patientenschulungen und verhaltenstherapeutische Maßnahmen zum Einsatz („Blasendrill“). Beim Kontinenztraining wird zwischen aktivem Miktionstraining, bei dem der Patient den selbständigen Umgang mit der Erkrankung lernt, und passivem Toilettentraining unterschieden. So kann bei pflegebedürftigen Patienten in einigen Fällen auch durch das Pflegepersonal ein Blasentraining vorgenommen werden. Für derartige Schulungen sind kurzfristige Besserungsraten bis knapp $90 \%$ beschrieben (15). Die längerfristigen Erfolge hängen stark von der Weiterbetreuung der Patienten ab und sind oft schlechter.

\section{Biofeedback}

Biofeedback ermöglicht dem Patienten unbewusste Vorgänge durch optische, akustische oder taktile Rückmeldung bewusst zu trainieren, möglicherweise auch wieder unterbewusst zu steuern. Ziel ist es, Blasenkontraktionen aktiv zu unterdrücken. Die therapeutische Wertigkeit des Verfahrens für die Dranginkontinenz im Gegensatz zur Stressinkontinenz ist noch nicht hinreichend untersucht.

\section{Elektrotherapie}

Zahlreiche Überlegungen rechtfertigen eine Inkontinenztherapie mit Elektrostimulation: Stärkung der Muskulatur des Beckenbodens, Unterstützung der proximalen Urethra, Unterdrückung von reflektorischen Detrusorkontraktionen und Einflussnahme auf Vaskularisierung und Durchblutung des Blasenhalses (4). Zahlreiche Methoden und qualitativ unterschiedliche Untersuchungen machen die Bewertung der Verfahren für die Dranginkontinenz schwierig. Hier sollte bei Versagen der konventionellen Therapie im Einzelfall ein Therapieversuch gestartet werden: Ein Ziel der Therapie kann auch sein, Abläufe wie Kontraktionen, Relaxationen ähnlich wie beim Biofeedback bewusst zu machen.

\section{Medikamentöse Therapie}

Reichen die beschriebenen Maßnahmen nicht aus, sind Medikamente auch in Kombination mit diesen Verfahren indiziert. Die Substanzen (Tab.1) stammen v.a. aus der Gruppe der Parasympa- thikolytika und vermindern (unwillkürliche) Detrusorkontraktionen durch Hemmung des vegetativen Nervensystems (Blockade von Muskarinrezeptoren). Die Folge sind seltenere Entleerungen der Blase mit größeren Urinvolumina. Ein bedeutender Begleiteffekt ist die Steigerung der Blasenkapazität. In Kombination mit Blasentraining sind größere Abstände zwischen den Miktionen zu erzielen. Unerwünschte Wirkungen der Präparate beruhen meist auf ihrem anticholinergen Wirkmechanismus, der nicht spezifisch auf die Blase beschränkt ist: Mundtrockenheit, Hypohidrosis, Obstipation, Übelkeit, aber auch Tachykardien sind typisch. Bei Glaukom sind die Präparate kontraindiziert bzw. nur in Kooperation mit einem erfahrenen Augenarzt einzusetzen. In einem aktuellen systematischen Review der Cochrane-Gruppe fand sich eine deutliche größere Blasenkapazität, eine größere Urinmenge bei Miktion, eine Abnahme der Miktionsfrequenz und der Inkontinenz. Die Medikation wurde gut vertragen, Mundtrockenheit trat jedoch bei 32\% der Patienten auf. Die Steigerung der Restharnmenge war ohne klinische Relevanz (4 ml) (9). Retardierte Formen, die seit einiger Zeit auf dem Markt sind, scheinen weniger Nebenwirkungen zu haben. Wichtig ist eine einschleichende Dosierung, die beim alten Menschen an die Organfunktion anzupassen ist. Hohe Dosierungen sind wegen der unerwünschten Wirkungen zu vermeiden. Bei Komedikation mit ebenfalls anticholinerg wirkenden Medikamenten ist besondere Vorsicht geboten, z.B. bei tri- und tetrazyklischen Antidepressiva, Neuroleptika, Antihistaminika, Metixen (Antiparkinsonmittel), Biperiden und Atropinderivaten (COPD). Zu Beginn der Therapie sind sonographische Restharnkontrollen erforderlich. Bei unkritischem Gebrauch führt nur ein knappes Drittel der Patienten die Medikation fort (12).

Eine weitere Behandlungsmöglichkeit ist die intravesikale Instillation von $5 \mathrm{mg}$ Oxybutynin in Kochsalzlösung, zweimal pro Tag für jeweils $30 \mathrm{~min}$. Dieses Verfahren kommt vorwiegend für Patienten in Betracht, die sich selbst katheterisieren. Vorteile gegenüber der systemischen Gabe sind nicht sicher nachgewiesen. Ist eine Indikation für eine antidepressive Therapie gegeben, so eignen sich trizyklische Antidepressiva aufgrund ihrer kombinierten Wirkung auf Detrusor und Sphinkter besonders gut bei gemischter Inkontinenz. Calciumantagonisten sollen bei Detrusorhyperreflexie ebenfalls den ge- 
häuften Harndrang herabsetzen und die Blasenkapazität erhöhen. Sie sind zur primären Therapie jedoch ungeeignet.

kurzgefasst: Die Stressinkontinenz wird durch Kombination aus Physiotherapie und Hilfsmittelversorgung therapiert. Bei unzureichendem Therapieerfolg stehen heute mehrere erprobte Operationsverfahren zur Verfügung. Die Dranginkontinenz kann durch Verhaltenstraining gut beeinflusst werden. Meist ist eine begleitende Medikation mit einem Anticholinergikum erfolgversprechend.

\section{Therapie der Überlaufinkontinenz}

\section{Akutintervention}

Die Therapie der Überlaufinkontinenz besteht zunächst in der Entleerung der übervollen Blase durch eine instrumentelle Harnableitung. Welches Verfahren dann gewählt wird (Einmal-Katheterismus oder ein anderes Ableitungsverfahren), hängt von Ursachenkomplex und Patientensituation ab. Bei obstruktiven Blasenentleerungsstörungen ist eine operative Sanierung der entsprechenden Auslasshindernisse vorrangig. Die Behandlung einer neurogenen Störung mit Detrusorakontraktilität (z.B. im Rahmen eines Diabetes mellitus) ist oft schwieriger. Auch hier kommen Trainingsmöglichkeiten mit Unterstützung durch Biofeedback-Maßnahmen in Betracht (2).

\section{Harndauerableitung}

Ist bei dieser Form der Inkontinenz der Einsatz einer instrumentellen Harnableitung im Sinne eines Dauerkatheters indiziert, sollte man den suprapubischen Katheter dem transurethralen Dauerkatheter vorziehen. Denn der Fremdkörper in der Harnröhre, insbesondere bei Frauen die Nähe zu Anus und Scheidenöffnung, erleichtert die Bakterienaszension. Unter suprapubischer Ableitung kann im Rahmen der Blasenrehabilitation ein Wasserlassen durch die Harnröhre trainiert werden. Harnröhrenstrikturen werden vermieden, es findet sich keine postinstrumentelle Urethritis, Prostatitis oder Epidydimitis und es kommt seltener zu kathetervermittelten problematischen Harnwegsinfektionen (2). Außerdem sollten Möglichkeiten der Beinbeutel- oder Hüftbeutelableitung für den verbesserten Tragekomfort bedacht werden.

\section{Therapie der Reflexinkontinenz}

Die Ziele bei der Therapie der Reflexinkontinenz sind vor allem:

- Herstellung einer ausreichenden Speicherfunktion

- Harnkontinenz oder adäquate Inkontinenzversorgung

- Restharnarme Entleerung unter tolerablem Druck in der Blase, Vermeidung von Druckschäden

Für die Reflexinkontinenz konnte nachgewiesen werden, dass die Therapie mit einem Blasenverweilkatheter mit einer hohen Komplikationsrate und verkürzter Lebenserwartung einhergeht. Deshalb sind die folgenden Therapieverfahren vorzuziehen: kurzgefasst: Eine Überlaufinkontinenz bedarf häufig einer Harndauerableitung, wenn die Therapieoptionen zur Behebung einer Obstruktion des unteren Harntraktes ausgeschöpft sind. Ein suprapubischer Blasenkatheter ist einem transurethralen Katheter meist vorzuziehen. Blasenverweilkatheter dienen nicht zur Erleichterung der Pflege, sondern sind ein invasives Harnableitungsverfahren mit zahlreichen Nebenwirkungen.

Ist keine Heilung möglich, sollten bei Harninkontinenz mit den zur Verfügung stehenden Behandlungsmöglichkeiten zumindest die Symptome deutlich reduziert werden. So können Folgeprobleme wie Pflegebedürftigkeit und soziale Isolation verhindert werden.

Autorenerklärung: Die Autoren erklären, dass sie keine finanziellen Verbindungen mit einer Firma haben, deren Produkt in dem Artikel eine wichtige Rolle spielt (oder mit einer Firma, die ein Konkurrenzprodukt vertreibt).

\section{Literatur}

1 Andersson KE, Chapple CR. Oxybutynin and the overactive bladder. World J Urol 2001; 19: 319-323

Bach D. Kathetermaterialien, Katheteraufbau und Kathetertypen. Jungjohann, Neckarsulm, In: Bach, D.; Brühl, P. (Hrsg): Harnwegsinfektionen. 1995: 8-15

3 Carr LK, Walsh PJ, Abraham VE, Webster GD. Favorable outcome of pubovaginal slings for geriatric women with stress incontinence. J Urol 1997; 157: 125-128

4 Engelmann H, Sundberg J. Harninkontinenz. De Gruyter, Berlin, In: Fischer W, Köbel H (Hrsg): Urogynäkologie in Praxis und Klinik. 1995

5 Fall M, Lindstrom S. Functional electrical stimulation: physiological basis and clinical principles. Int Urogynecol J 1994; 5: 296-304

6 Fantl JA, Newman DK, Colling J et al. Urinary Incontinence in Adults: Acute and Chronic Management. Public Health Service, Agency for Health Care Policy and Research, Clinical Practice Guideline, No. 2. Rockville: Department of Health and Human Services. 1996

Fusgen I. Harninkontinenz im Alter. Urologe A 1991; 30: 235-238

8 Fusgen I, Hauri D. Trospium chloride: an effective option for medical treatment of bladder overactivity. Int J Clin Pharmacol Ther 2000; 38: 223-234

9 Hay-Smith EJ, Bo Berghmans LC, Hendriks HJ et al. Pelvic floor muscle training for urinary incontinence in women. Cochrane Database Syst Rev 2001: CD001407

10 Hay-Smith J, Herbison P, Ellis G, Moore K. Anticholinergic drugs versus placebo for overactive bladder syndrome in adults. Cochrane Database Syst Rev 2002; 3: CD003781

11 Junemann KP, Hoang-Bohm J, Wipfler G et al. Aktueller Therapiestandard in der Behandlung der genuinen Streßinkontinenz der Frau mittels Harnröhren-Mini-Devices. Urologe A 1997; 36: 405-412

12 Kennedy A. Bladder re-education for the promotion of continence. London: Prentice Hall, In: Roe, B (ed): Clinical Nursing Practice: The Promotion and Management of Continence. 1992: 77-93

13 Lawrence M, Guay DR, Benson SR, Anderson MJ. Immediate-release oxybutynin versus tolterodine in detrusor overactivity: a population analysis. Pharmacotherapy 2000; 20: 470-475

14 Pannek J, Haupt G, Sommerfeld HJ et al. Urodynamic and rectomanometric findings in urinary incontinence. Scand J Urol Nephrol 1996; 30: 457-460

15 Schwenzer T. Derzeitiger Stand der medikamentösen Beeinflussbarkeit des unteren Harntraktes. Gynakologe 1992; 25: 247-257

16 Sogbein SK, Awad SA. Behavioural treatment of urinary incontinence in geriatric patients. Can Med Assoc J 1982; 127: 863-864

17 Thüroff JW, Casper F, Heidler H. Pelvic floor stress response: effect on periurethral muscle contraction and pelvic floor tone on substitute urethra. Neurourol Urodyn 1987; 6: 153

18 Ulmsten U, Falconer C, Johnson P et al. A multicenter study of tension-free vaginal tape (TVT) for surgical treatment of stress urinary incontinence. Int Urogynecol J Pelvic Floor Dysfunct 1998; 9: 210-213

19 van Kerrebroeck PHEV. Long-term tolerability and effcacy of once-daily (OD) tolterodine in the treatment of overactive bladder (OAB). Int Urogynecol J 2001; 12 (Suppl 3); 549

- Getriggerte Reflexentleerung

- Intermittierender Selbstkatheterismus, ggf. in Kombination mit anticholinerger Therapie (sinnvoll bei geringem Entleerungsvolumen und Inkontinenz)

- Enterozystoplastik bei stark gestörter Dehnbarkeit der Blase 